\title{
Balkanologie
}

Balkanologie Revue d'études pluridisciplinaires

Vol. XII, $n^{\circ} 1$ | 2010

Volume XII Numéro 1

\section{Stevan Moljević et la question nationale serbe}

\section{Yves Tomić}

\section{(2) OpenEdition}

Journals

Édition électronique

URL : https://journals.openedition.org/balkanologie/2144

DOI : 10.4000/balkanologie.2144

ISSN : 1965-0582

\section{Éditeur}

Association française d'études sur les Balkans (Afebalk)

\section{Référence électronique}

Yves Tomić, «Stevan Moljević et la question nationale serbe », Balkanologie [En ligne], Vol. XII, $n^{\circ} 1$ । 2010, mis en ligne le 15 février 2013, consulté le 28 juin 2022. URL : http://journals.openedition.org/ balkanologie/2144; DOI : https://doi.org/10.4000/balkanologie.2144

Ce document a été généré automatiquement le 17 décembre 2020.

(c) Tous droits réservés 


\title{
Stevan Moljević et la question nationale serbe
}

\author{
Yves Tomić
}

\section{Introduction}

1 Alors que la version communiste des événements de la Seconde Guerre mondiale avait prédominé entre 1945 et 1990, de nombreux écrits sont venus depuis apporter une autre lumière sur cette période tragique de l'histoire des peuples yougoslaves ${ }^{1}$. Pour autant, cela ne signifie pas qu'une approche plus mesurée et distanciée a succédé à la vision idéologisée qui avait cours précédemment. En Serbie, de nombreux ouvrages sur le mouvement de la Ravna Gora ont été publiés depuis le début des années $1990^{2}$. Les ouvrages relatant l'épopée des partisans communistes ont été remplacés par des livres apologétiques sur le mouvement que dirigea Draža Mihailović pendant la Seconde Guerre mondiale ${ }^{3}$. Parmi toute cette production éditoriale, force est de constater que certains acteurs pourtant significatifs ont été oubliés. C'est le cas en particulier de Stevan Moljević, membre du Comité central national (Centralni nacionalni komitet, CNK), structure dont s'était doté le mouvement de la Ravna Gora pour traiter des questions politiques et de la propagande.

2 Stevan Moljević est principalement connu pour être l'auteur, au printemps 1941, d'un memorandum préconisant la création d'une Grande Serbie dans une Grande Yougoslavie. Dans ce document, il prévoit l'expulsion des non-Serbes habitant les territoires devant intégrer l'entité serbe et des échanges de population, en particulier entre Serbes et Croates. Son texte est cité dans la plupart des ouvrages dénonçant le nationalisme serbe ou abordant la question du nettoyage ethnique ${ }^{4}$. Pour autant, le parcours de Stevan Moljević demeure peu connu ${ }^{5}$. Nous présenterons dans le cadre de cet article le cheminent intellectuel d'un des principaux acteurs de la vie intellectuelle à Banja Luka pendant l'entre-deux-guerres. L'analyse de son parcours constitue une contribution à l'étude de l'engagement politique des intellectuels serbes entre 1918 et 1941, ainsi que pendant la Seconde Guerre mondiale. Nous nous intéresserons 
également à l'émergence du Club culturel serbe à fin des années 1930 auquel Stevan Moljević adhérera en 1939 et dont les membres éminents joueront un rôle important pendant la Seconde Guerre mondiale tant au sein du gouvernement yougoslave en exil qu'auprès du commandement suprême de "l'Armée yougoslave dans la patrie " (Jugoslovenska vojska u otadžbini, JVuO) que Stevan Moljević accompagnera entre 1942 et $1945^{6}$.

\section{Stevan Moljević, notable et intellectuel de Banja Luka}

Fils de paysans, Stevan Moljević est né à Rudo (Bosnie-Herzégovine) en 1888. Il fréquente l'école primaire de son village, puis parvient à s'inscrire au lycée à Sarajevo en 1906. Il obtient une bourse afin d'étudier le droit à Zagreb. Il y poursuit ses études jusqu'à l'obtention de son doctorat. Pendant ses années de lycée à Sarajevo, il intègre une organisation secrète de jeunes lycéens dirigée par Vladimir Gaćinović, contestant l'autorité de l'Autriche-Hongrie sur la Bosnie-Herzégovine ${ }^{7}$. L'organisation en question prend une orientation révolutionnaire après l'annexion de la Bosnie-Herzégovine par l'Autriche-Hongrie en 1908. Stevan Moljević aide Bogdan Žerajić dans la préparation d'un attentat contre le gouverneur de la Bosnie-Herzégovine, le général Marijan Varešanin à l'occasion de la visite de l'empereur François-Joseph à Sarajevo fin maidébut juin $1910^{8}$.

Plus tard, Stevan Moljević écrira à propos de cet événement :

Nous avons vécu la visite de l'empereur en Bosnie-Herzégovine comme une provocation irrévérencieuse pour nous les Serbes.Par cette visite, les dirigeants autrichiens ont voulu dire au monde, et plus particulièrement aux Serbes, que l'Autriche avait réglé pour toujours la question de la Bosnie et de l'Herzégovine qui par l'annexion sont devenues des parties à part entière et indivisibles du puissant empire, que tous les espoirs et les aspirations des Serbes et de la Serbie pour la Bosnie étaient enterrés à jamais 9 .

5 L'attentat échoue et Žerajić se donne la mort le 15 juin 1910. À la fin de ses études, en 1913, Moljević s'installe à Banja Luka où il organise la société nationale Soko (Faucon) ${ }^{10}$. En 1915, il est arrêté par les autorités autrichiennes parmi de nombreux notables serbes. Il sera accusé avec 155 autres personnes de haute trahison et d'avoir soutenu la cause grand-serbe, à savoir l'unification de la Bosnie-Herzégovine à la Serbie. Toutefois, en l'absence de preuves, il est libéré et envoyé sur le front italien jusqu'à la fin de la guerre $^{11}$.

6 Après la Première Guerre mondiale, Stevan Moljević devient avocat à Banja Luka et refonde la société Soko avec pour mission la diffusion du sentiment national serbe auprès de la jeunesse. Il travaille également à la diffusion de la culture française à Banja Luka et dans la région de la Bosanska Krajina. En 1922, il fonde le club yougolavofrançais (ou Cercle franco-yougoslave dans les documents officiels français) dont il sera le président pendant 17 ans. Le club organise des cours de français pour les jeunes et les adultes. Il dispose également d'un jardin pour enfants pour lequel une enseignante est employée. En 1939, la bibliothèque du club compte 1600 fascicules et met à la disposition des lecteurs une quinzaine de périodiques dans sa salle de lecture ${ }^{12}$. Une dizaine d'enseignants assure les cours de français. Le club a été installé dans la grande maison de Stevan Moljević ${ }^{13}$. Pour ses activités, il reçoit l'aide matérielle de la France. Stevan Moljević fonde un club similaire à Prijedor avec l'appui de l'ambassade de France $^{14}$. Stevan Moljević n'est pas le simple administrateur du club : à l'occasion du 
quarantième anniversaire de la parution de l'article «J'accuse » d'Emile Zola, il tient une conférence, le jeudi 13 janvier 1937, sur l'affaire Dreyfus et le procès contre Zola ${ }^{15}$. Ses activités ne passent pas inaperçues de l'ambassade de France en Yougoslavie : en août 1937, il est nommé chevalier de la Légion d'honneur. Stevan Moljević se montre très dynamique et sur le modèle du club yougoslavo-français, il fonde le club yougoslavo-britannique dont il sera le président pendant 5 ans.

7 Stevan Moljević est actif dans plusieurs publications de Banja Luka. Dans la revue Razvitak (Le Développement), il est membre du comité de rédaction et responsable de la rubrique politique. Relancée en janvier 1935, la revue avait été fondée en 1910 par Petar Kočić, figure politique importante de la Bosanska Krajina. En 1936, il relance le journal Otadžbina (La Patrie), fondé également par Petar Kočić en 1907. Le premier numéro paraît le 21 novembre 1936. Il en devient le rédacteur en chef. Le journal se veut indépendant des partis politiques et soutient les valeurs de la démocratie, alors que les dictatures se multiplient en Europe ${ }^{16}$. Il s'oppose ouvertement au fascisme, ce qui suscitera des plaintes de l'ambassade du Reich allemand à Belgrade. Otadžbina se prononce également pour la décentralisation de la Yougoslavie et l'autonomie des communautés locales. De fait, le journal défend les intérêts de Banja Luka et de la Bosanska Krajina et aspire à sortir cette région du sous-développement.

Stevan Moljević se pose en défenseur des intérêts serbes en Bosnie-Herzégovine et s'oppose au bošnjakluk, idéologie fondée par Benjamin Kállay, gouverneur de la BosnieHerzégovine dans les années 1880, dans le but d'entraver et d'étouffer les consciences nationales serbe et croate. En 1937, alors que l'Organisation musulmane yougoslave (JMO) participe au pouvoir dans un gouvernement de coalition dirigé par Milan Stojadinović, il dénonce l'état d'esprit antiserbe qui prédomine, selon lui, dans la population musulmane.

\section{Stevan Moljević et le Club culturel serbe}

Stevan Moljevic a été membre du Parti démocrate indépendant (Samostalna demokratska stranka) fondé par Svetozar Pribićević. Toutefois, nous ne disposons pas d'éléments sur son engagement et ses activités éventuelles au sein de cette organisation. Par contre, son implication au sein du Club culturel serbe à la fin des années 1930 est mieux connue. Afin de mieux situer les idées de Stevan Moljević, il convient de présenter plus en détail le Club culture serbe qui rassemble à la fin des années 1930 des intellectuels serbes de renom qui joueront pour certains un rôle politique non-négligeable pendant la Seconde Guerre mondiale.

Les autorités politiques yougoslaves des années 1920 à la fin des années 1930 légitiment leur pouvoir sur la base du yougoslavisme. À aucun moment, elles ne font référence à une idéologie grand-serbe. Cette politique est également contestée par des intellectuels serbes, souvent membres de partis politiques ${ }^{17}$. Ces derniers se prononcent, dans les années 1920 , pour une solution intermédiaire entre le centralisme et le fédéralisme ${ }^{18}$. Toutefois, la plupart des intellectuels serbes soutiennent, souvent avec ferveur, l'idée de l'unité nationale des Serbes, Croates et Slovènes. Néanmoins, à partir de 1937 une partie de des élites intellectuelle et économique serbe se regroupe afin de défendre les intérêts serbes dans la Yougoslavie, en premier lieu en Bosnie et en Croatie ${ }^{19}$. Le Club culturel serbe (Srpski kulturni klub, SKK) est fondé en janvier 1937 pour être un lieu de discussion sur les questions relatives à la culture nationale serbe, la culture étant 
entendue dans un sens large (spirituelle et matérielle) ${ }^{20}$. Lors de son assemblée fondatrice, le 4 février 1937, le Club compte 70 membres dont 22 enseignants de l'Université de Belgrade ainsi que d'autres facultés du pays. D'anciens ministres, des officiers en retraite, des industriels, des banquiers, des avocats, etc. font également partie des membres fondateurs de cette organisation. L'assemblée fondatrice élit l'historien Slobodan Jovanović comme président, l'avocat Nikola Stojadinović et l'écrivain et avocat Dragiša Vasić comme vice-présidents, Vaso Čubrilović, chargé de cours à l'Université de Belgrade, comme secrétaire.

11 Le Club culturel serbe est créé par des intellectuels estimant que les autorités yougoslaves ne sont pas en mesure de protéger les intérêts nationaux serbes, en particulier dans le sud (Macédoine et Kosovo) et le nord-ouest du pays (Bosnie, Croatie). Il prévoit d'étendre son influence dans les « régions frontalières » (granične oblasti) où les Serbes «sont menacés par les influences étrangères " ${ }^{21}$. Si la création du Royaume des Serbes, Croates et Slovènes a été perçue comme une solution à la question nationale serbe, les membres du SKK constatent que la nation serbe n'est pas intégrée sur les plans national, culturel et économique. Le SKK crée des sous-comités en particulier dans les régions mixtes sur le plan national: Voïvodine, Serbie du sud (Macédoine, Kosovo), Bosnie et Herzégovine. Il s'agit pour les sous-comités du SKK de renforcer la conscience nationale serbe dans les régions où les Serbes sont mélangés à d'autres nationalités et d'affirmer le caractère serbe de la Voïvodine, de la Bosnie-Herzégovine ou encore de la Macédoine. En 1939, le SKK fonde un journal sous le nom de Srpski glas afin de diffuser ses idées : le slogan en est « une Serbité forte - une Yougoslavie forte » qui annonce le programme du mouvement de la Ravna Gora pendant la Seconde Guerre mondiale (« Une Grande Serbie dans une Grande Yougoslavie ») 22.

12 L'écrivain Dragiša Vasić en dirige la rédaction. Le Club organise par ailleurs des tribunes où les questions relatives à la position des Serbes dans la Yougoslavie sont abordées, mais aussi les questions relatives à l'éducation, à l'économie et à la situation internationale ${ }^{23}$. Bien que nombre de ses membres se soient formés à l'étranger, le SKK rejette les influences étrangères dans la culture serbe (les courants modernistes comme le dadaïsme, le surréalisme, le cubisme, le futurisme sont condamnés; dans le domaine des sciences sociales et humaines, les modèles étrangers comme le marxisme sont dénoncés). Il préconise un retour aux traditions et aux normes de la société serbe d'avant-guerre, la culture devant reposer sur les valeurs du fondateur de l'Église orthodoxe serbe, Saint Sava ${ }^{24}$.

13 Dans le contexte du règlement de la question nationale croate en 1939 dans un sens fédéral, les activités du Club culturel serbe prennent une dimension largement politique. Toutes ses activités sont désormais orientées vers le règlement de la question nationale serbe à l'intérieur de la Yougoslavie. Le SKK apparaît clairement comme le défenseur des intérêts serbes en Yougoslavie. Alors qu'un accord est en discussion entre l'opposition croate et le gouvernement yougoslave sur la création d'une unité territoriale croate au sein de l'État, le Club culturel serbe réagit vivement et avertit le gouvernement des risques encourus. Ses dirigeants contestent les frontières et les prérogatives de la nouvelle entité croate qui se profile. Le 30 juin 1939, Stevan Moljević tient une conférence sur la Banovine du Vrbas au cours de laquelle il explique que la question croate ne doit pas être réglée en ouvrant la question serbe. Or, selon lui, la question serbe sera ouverte si des territoires peuplés de Serbes (Bosanska Krajina, Banija, Kordun, Lika et Dalmatie du nord) doivent intégrer l'entité croate $^{25}$. Son 
intervention intitulée «Uloga i značaj Vrbaske banovine » [Le rôle et l'importance de la banovine du Vrbas] fait forte impression auprès des membres du SKK. Aussi, la décision est prise de fonder un comité à Banja Luka. Ce dernier est créé en décembre 1939 et Stevan Moljević en devient le responsable. La crainte de Stevan Moljević est de voir la Bosanska Krajina sacrifiée par Belgrade au profit de la banovine de Croatie ${ }^{26}$. Au lendemain de la conclusion de l'accord du 26 août 1939 qui établit la banovine de Croatie, le Club culturel serbe réagit fermement et conteste les limites de la nouvelle entité $^{27}$. Il estime que les représentants politiques des Serbes n'ont pas été consultés. Il refuse de laisser des districts peuplés majoritairement de Serbes à la Banovine de Croatie, perçue comme la première étape de la création d'une Grande Croatie :

Notre point de vue est clair. Nous voulons un accord, mais basé sur certains principes : soit ethniques, soit historiques ou soit géographiques et économiques. Mais pour tout le territoire sur lequel vivent les Serbes et les Croates. Nous ne voudrons jamais laisser des districts avec une majorité serbe dans les frontières de la Croatie, de la Dalmatie, de la Bosnie et de la Slavonie à la Banovine de Croatie. En demandant la révision du prétendu accord, nous souhaitons que l'on donne aux Serbes vivant dans les frontières de l'ancienne Croatie et Slavonie le plein droit de se prononcer librement pour savoir si leurs districts resteront en Croatie ou seront associés à l'unité serbe ${ }^{28}$.

14 En réaction à l'accord, le club culturel serbe réclame la formation d'une unité administrative et politique serbe.

J'ai un conseil que je pense pouvoir donner aux Serbes en tant que Serbe moimême. Nous, Serbes, devons comprendre que nous avons une double tâche à accomplir. En premier lieu, nous devons défendre la Serbité. Quand on définit les contours d'une unité ethnique croate, il faut alors inévitablement définir les contours d'une unité ethnique serbe. Il serait idiot d'affirmer que seuls les Croates ont une conscience nationale dans cet Etat. Et qu'eux seuls ont une histoire, tandis que les Serbes n'auraient pas de conscience nationale ni d'histoire, mais représenteraient une masse amorphe malléable à merci. En même temps que la question croate a été ouverte, la question serbe l'a été également et les Serbes doivent défendre en unissant leurs forces ce qui leur appartient ${ }^{29}$.

15 Les comités du SKK des villes de Vukovar, Vinkovci et Dalj, situées dans la région du Srem occidental, exigent que les territoires de ces communes, où les Serbes représentent un cinquième de la population, soient rattachés à la future unité serbe. Le SKK relance le projet d'intégration nationale de l'ensemble des Serbes dans une même structure étatique L'idéologie yougoslave intégrale est sévèrement critiquée et dénoncée, mais le SKK demeure attaché à l'idée d'un État yougoslave. Selon Slobodan Jovanović, l'unification forcée des Serbes et des Croates a été contre-productive et la combinaison entre unitarisme et centralisme a envenimé leurs relations. Ses dirigeants exigent l'incorporation de certaines régions de la Banovine croate peuplées de Serbes, ainsi que de la Bosnie et de l'Herzégovine à la future entité serbe ${ }^{30}$. Un projet d'établissement d'une unité territoriale serbe est élaboré en 1940 par le gouvernement yougoslave. Il prévoit l'unification des banovines du Vrbas, de la Drina, du Danube, de la Morava, de la Zeta et du Vardar dans une entité dénommée "pays serbe » (Srpska zemlja) dont le centre serait Skopje, capitale actuelle de la Macédoine. Certaines villes situées dans l'unité croate devraient lui être intégrées (Brčko, Travnik, Fojnica). Cependant, la réorganisation du royaume yougoslave sur une base fédéraliste et ethnique est entravée par l'arrivée de la Seconde Guerre mondiale.

16 Les membres du SKK réfléchissent également à la solution du problème des minorités nationales qui remettent en cause l'assise de la future entité serbe. Les minorités 
nationales représentent $17 \%$ de la population selon le recensement de 1921. Elles sont particulièrement nombreuses en Voïvodine (60\% de la population) et en Serbie du sud (Kosovo $-40 \%$ de la population). Certains territoires de ces régions étant peuplés densément par des minorités nationales, il convient, selon les intellectuels du SKK, de les nationaliser, à savoir d'y renforcer les éléments serbes. Dans la plupart des réflexions sur cette problématique, la solution préconisée est le déplacement des minorités nationales, la politique de colonisation du Kosovo menée par les autorités yougoslaves s'étant avérée inefficace pour changer la structure de la population du sud de la Serbie. La minorité albanaise, dont certaines zones de peuplement coupent des zones de peuplement serbe, est particulièrement visée. Selon les chiffres de 1921, les Albanais du Kosovo représentaient $66 \%$ de la population de cette région contre $25 \%$ de Serbes. Lors d'une conférence du SKK tenue le 7 mars 1937, Vaso Čubrilović préconise le déplacement massif des Albanais du Kosovo par l'usage de moyens coercitifs. Les Albanais sont perçus comme une menace politique et nationale car ils constituent une population compacte brisant la continuité des zones de peuplement serbe :

Il est impossible de refouler les Albanais par la seule colonisation graduelle; c'est le seul peuple qui soit parvenu au cours de notre chemin millénaire non seulement à résister au noyau de notre État, Raška et Zeta, mais même à nous causer du tort, en repoussant nos frontières ethniques vers le nord et l'est. Alors que nos frontières ethniques se sont déplacées, au cours du dernier millénaire, au nord jusqu'à Subotica, et au nord-ouest jusqu'à Kupa, les Albanais nous ont chassés de la région de Skadar, ancienne capitale de Bodin, de Metohija et du Kosovo. La seule manière et le seul moyen de les refouler est la force brutale d'un pouvoir d'État organisé, au sein duquel nous les avons toujours dominés. (... ${ }^{31}$

Vaso Čubrilović précise quels doivent être les districts à évacuer et décrit le processus de repeuplement de ces régions par des colons issus du Monténégro, d'Herzégovine, de la Lika et de la Krajina. Les propositions de Vaso Čubrilović ne sont pas isolées et rejoignent celles avancées par Djoko Perin en juin 1937. Ce dernier en effet suggère de transférer une partie des Albanais du Kosovo vers l'Albanie et de déplacer dans d'autres régions de Yougoslavie la plupart d'entre eux afin que la population serbe devienne largement majoritaire dans cette région ${ }^{32}$.

18 La Voïvodine, région la plus riche du pays sur laquelle s'appuie la capitale Belgrade, constitue également un objet de préoccupation pour les membres du SKK dans la mesure où les Serbes n'y sont pas majoritaires (474 000 personnes, soit 32\% de la population en 1936) et que la minorité hongroise adossée à la Hongrie (392000 personnes, soit $26,5 \%$ de la population en 1936) y est importante, tout comme la minorité allemande $(338000,23 \%)^{33}$. Afin de renforcer la présence serbe en Voïvodine, il est proposé des échanges forcés de population plutôt qu'une colonisation de la province qui serait difficile à mettre en œuvre. En effet, pour que les Serbes y deviennent majoritaires, il aurait fallu y installer plus de 523000 colons serbes et plus d'un million pour qu'ils représentent $60 \%$ de la population. Selon le SKK, les populations hongroise, allemande et bunjevac ${ }^{34}$ pourraient s'installer en Slavonie, région que 200000 Serbes quitteraient pour aller en Voïvodine. Ces considérations sur les minorités nationales montrent que le SKK ne souhaitait pas uniquement fixer les limites d'une unité (fédérale) serbe à l'intérieur de la Yougoslavie, mais également assurer l'homogénéité de sa population en renforçant le poids démographique des Serbes par des déplacements forcés des populations non-serbes minoritaires ou des échanges de population. Qu'il soit question du Kosovo ou de la Voïvodine, les échanges de population entre la Turquie et la Grèce en 1921-1922 sont le principal point de 
référence pour appuyer la solution de la question des minorités nationales. Les débats menés au sein du Club culturel serbe à la fin des années 1930 marquent un tournant dans la formulation de l'idéologie grand-serbe, dans la mesure où les transferts forcés de population deviennent clairement le moyen de créer une entité étatique la plus homogène possible. Au XIXème siècle, les dirigeants serbes ne raisonnaient pas dans ces catégories.

Le Club culturel serbe contribue à renforcer la conscience nationale serbe à l'intérieur de la Yougoslavie à la fin des années 1930. Dans les publications et les discussions prédomine l'idée que là où se trouvent des Serbes, là se trouve la Serbie. Ses membres soulignent le caractère serbe de la Voïvodine, de la Bosnie, de l'Herzégovine, de la Slavonie, de la Baranja, du Srem occidental ainsi que de la Macédoine ${ }^{35}$.

\section{Stevan Moljević dans la guerre}

20 En avril 1941, le royaume de Yougoslavie s'effondre après avoir été envahi par les forces de l'Axe. Le jour de la proclamation de l'État indépendant de Croatie (NDH), le 10 avril, Stevan Moljević prend le chemin de l'exode avec plusieurs de ses amis alors qu'il est âgé de 54 ans. Il sait qu'il sera la cible du nouvel ordre politique en train de s'installer dans la région. Il se réfugie au Monténégro où il arrive le 17 avril. Il réfléchit alors aux raisons de la disparition de la Yougoslavie et aux formes futures que pourrait prendre cet État. Il préconise l'établissement d'un État fédéral comprenant trois unités fédérales: Serbie, Croatie et Slovénie qui engloberaient chacune leur territoire ethnique. Il présente ses idées à quelques intellectuels, dont les professeurs de l'université de Belgrade Vasilj Popović et Vasa Čubrilović. Il consigne alors ses idées dans un texte qui paraîtra le 30 juin 1941 dans la ville monténégrine de Nikšić sous la forme d'une brochure intitulée « À propos de notre État et de ses frontières ».

21 Il ne s'agit donc pas au départ d'un document officiel du mouvement de la Ravna Gora de Draža Mihailović, mais son contenu sera largement repris dans les positions politiques de ce mouvement. Le texte de Stevan Moljević fixe comme objectif la création d'une Grande Serbie dans une Grande Yougoslavie transformée en un État fédéral comprenant trois unités (serbe, croate et slovène). Il s'agit d'unifier les territoires ethniques du peuple serbe dans un même cadre étatique. Afin de réaliser ce but, il prévoit l'expulsion des non-serbes habitant les territoires censés faire partie de l'entité serbe et des échanges de populations, en particulier entre Serbes et Croates.

(...) Pour cette raison, le premier et principal devoir s'imposant aux Serbes est :

qu'ils fondent et organisent une Serbie homogène qui doit englober tout l'espace ethnique sur lequel vivent les Serbes et lui assurent les lignes de communication et les points stratégiques nécessaires, ainsi que les régions économiques de telle sorte que soit rendue possible et assurée une vie culturelle, politique et économique libre et un développement pour tous les temps.

Ces lignes de communication et points stratégiques, nécessaires pour la sécurité, la vie et le maintien de la Serbie, quand bien même ils n'auraient pas de majorité serbe, doivent servir à la Serbie et au peuple serbe afin de ne pas voir se renouveler les graves souffrances que ses voisins lui infligeront dès que l'occasion se présentera.

Les déplacements et les changements de populations, plus particulièrement entre les Croates des territoires serbes et les Serbes des territoires croates, constituent la seule voie pour les délimiter et créer de meilleurs relations entre eux, et par conséquent supprimer toute possibilité que les crimes horribles qui se sont produits 
dans la guerre passée mais surtout au cours de la guerre actuelle ne se renouvellent, dans tout l'espace où les Serbes et les Croates sont mélangés et où les Croates et les Musulmans ont planifié d'exterminer les Serbes ${ }^{36}$.

Carte correspondant au projet de Stevan Moljević (juin 1941)

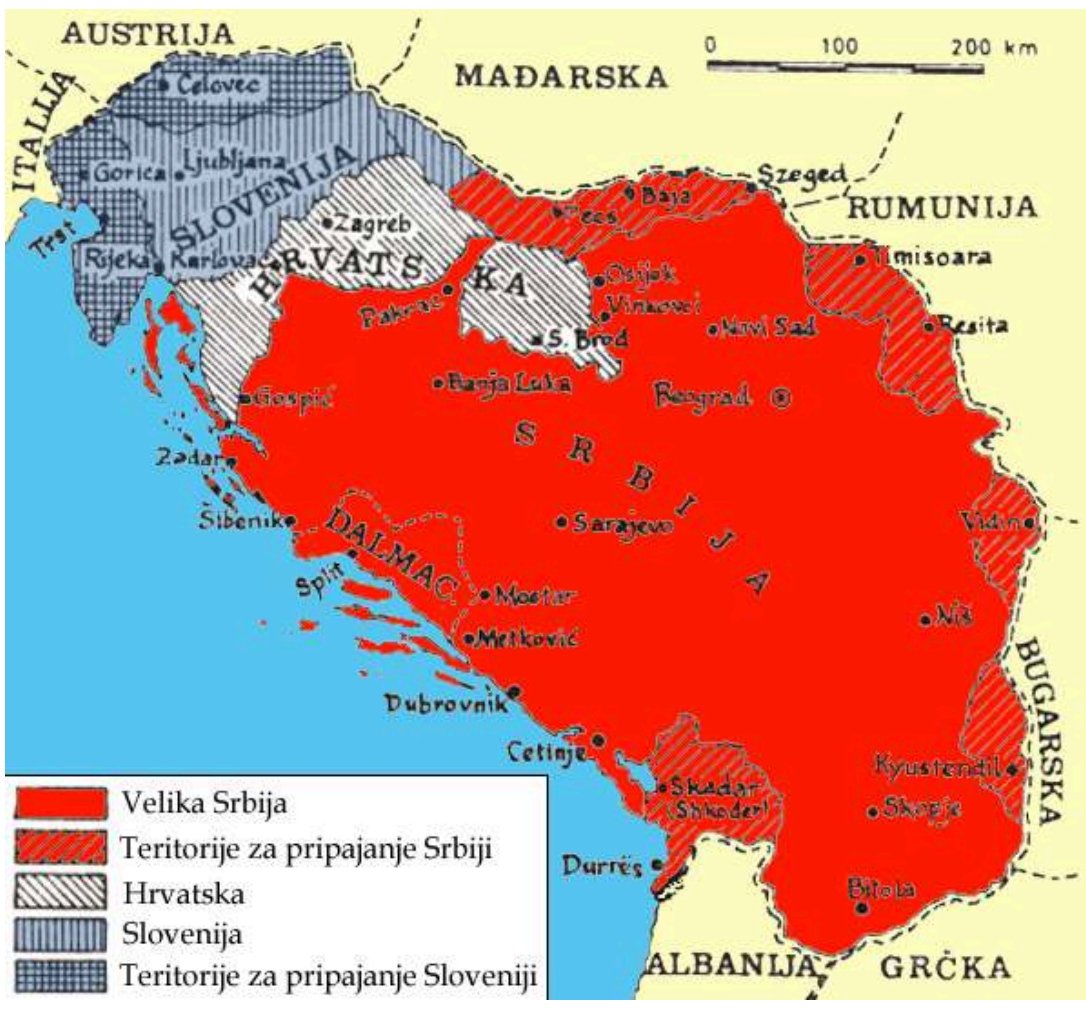

Source : http://en.wikipedia.org/wiki/Greater_Serbia

Selon Stevan Moljević, les autorités politiques serbes ont commis une grave erreur en 1918 en n'établissant pas les frontières de la Serbie à l'intérieur du Royaume des Serbes, Croates et Slovènes ${ }^{37}$. Il s'agit donc de réparer cette erreur en rassemblant tous les territoires peuplés de Serbes et en leur assurant une sortie sur la mer Adriatique. La Grande Serbie comprendrait donc à l'est et au sud-est la Serbie et la Serbie du Sud (Macédoine et Kosovo) auxquelles seraient agrégées les villes bulgares de Vidin et Kjustendil, au sud le Monténégro, l'Herzégovine et le nord de l'Albanie, à l'ouest la Bosnie, la Dalmatie du nord, la partie serbe de la Lika, le Kordun, la Banija et une partie de la Slavonie. Le littoral dalmate à partir de Šibenik en descendant vers le Monténégro appartiendrait au territoire serbe.

La Grande Yougoslavie serait construite sur une base fédérale : elle comprendrait de fait une Grande Serbie, une Croatie réduite et une Grande Slovénie. Stevan Moljević, s'inspirant probablement du Načertanije d'Ilija Garašanin, envisage également un rapprochement avec la Bulgarie. Selon lui, les Serbes étant les seuls à avoir sérieusement résisté aux Ottomans et aux Allemands, ont ainsi acquis le droit à diriger les Balkans. Pour réaliser leur mission historique, «les Serbes doivent avoir l'hégémonie dans les Balkans, et pour avoir l'hégémonie dans les Balkans, ils doivent préalablement avoir l'hégémonie en Yougoslavie ».

24 Il décide de proposer son aide au mouvement de Draža Mihailović à qui il adresse son projet de réorganisation de la Yougoslavie. En octobre 1941, il prend la route de la 
Serbie en passant par la localité de Plevlje, située dans la région du Sandžak, où il arrive le 21 octobre. Pendant son voyage, il a été blessé à la tête lors d'une attaque. Sa blessure a nécessité une opération par deux médecins. Il quitte Plevlje le 13 janvier 1942 pour son village natal, Rudo, d'où il poursuit son chemin pour la ville d'Užice qu'il atteint le 3 février 1942. N'étant pas en sécurité à Užice, il se réfugie à Požega le 3 avril 1942. C'est lors de son séjour dans cette localité qu'il rencontre Dragiša Vasić, l'ancien dirigeant du Club culturel serbe et rédacteur en chef du journal Srpski glas. Dragiša Vasić est l'un des principaux dirigeants politiques du mouvement de la Ravna Gora. Ils discutent de son projet et Stevan Moljević demande à rencontrer Draža Mihailović. Le 23 avril, il adresse un courrier à ce dernier dans l'attente de le rencontrer.

La rencontre se produit à Zlatar entre les 21 et 26 mai 1942. Stevan Moljević a l'occasion d'expliciter ses idées à Draža Mihailović. Il le sensibilise en particulier à la question de la Bosanska Krajina, région qui, selon lui, a été négligée par la classe politique serbe de l'entre-deux-guerres. Il envisage d'ailleurs l'unification de cette région à la Krajina croate (Banija, Kordun et Lika), ainsi qu'au nord de la Dalmatie dans le cadre d'une région spécifique qui aurait un débouché maritime autour de Šibenik ou Zadar ${ }^{38}$. Draža Mihailović approuve ses points de vue et Stevan Moljević intègre officiellement le mouvement bien qu'il ait été associé dès août 1941 au Comité central national. Mais ce n'est qu'à l'automne 1942, que Dragiša Vasić et Stevan Moljević rejoignent le Commandement suprême de l'Armée yougoslave dans la patrie (JVuO) à la demande de Draža Mihailović. Stevan Moljević a ainsi séjourné en Serbie du 3 février au 25 octobre 1942. Draža Mihailović souhaite renforcer la dimension politique du mouvement et développer un travail de propagande plus efficace. C'est à cette époque qu'est décidé le lancement d'un véritable organe du mouvement de la Ravna Gora. Le premier numéro du journal Ravna Gora est publié le $1^{\text {er }}$ février $1943^{39}$. Le travail politique et de propagande est confié aux trois anciens membres du Club culturel serbe que sont Dragiša Vasić, Stevan Moljević et Mladen Žujović. Le numéro du $1^{\mathrm{er}}$ mars 1943 précise le programme politique du mouvement de la Ravna Gora, qui correspond aux idées déjà formulées par Stevan Moljević en 1941 :
Nos aspirations sont claires et insoupçonnées. Nous souhaitons rassembler toutes les régions serbes dans une entité étroitement liée. Nous souhaitons que les Serbes après la guerre soient les maîtres incontestés dans leur demeure, qu'ils développent sans entrave leurs capacités intellectuelles, morales, nationales et leur aptitude au travail, sans intrus et autre élément non-serbe qui se sont introduits à la suite d'erreurs et de hasards historiques sur le sol national serbe. Et nos aspirations et ambitions politiques incontestées sont de garantir au peuple serbe, après la libération, la pleine liberté de son destin, la pleine démocratie et la justice sociale, ce qui figure dans son esprit et ses aspirations. Nous voulons créer en lui l'idéal du travail, de l'honnêteté et du sacrifice. Nous voulons le nettoyer de la racaille nationale, sociale et morale. En un mot, nous voulons le renouveau national, social et moral. Ce n'est qu'à travers une serbité purifiée, ses fondements moraux, nationaux et sociaux fermes, que nous pouvons compter sur une Yougoslavie forte, puissante et fiable, ainsi que sur des Balkans forts et indépendants. C'est là que se trouvent le sens et l'importance de notre combat difficile et sanglant débuté sur la Ravna Gora ${ }^{40}$.

Toutefois, quelques mois plus tard, les conseillers politiques du mouvement de la Ravna Gora se déchirent à propos du rôle de la Grande-Bretagne, Dragiša Vasić considérant les britanniques comme les fossoyeurs du peuple serbe contre l'avis de Stevan Moljević, lequel estime que leur mouvement doit s'appuyer sur les Anglais et les Américains ${ }^{41}$. Début juin 1943, alors que le Commandement suprême de la JVuO se réinstalle en 
Serbie, Dragiša Vasić abandonne le Comité central national (jusqu'en janvier 1944). Par ailleurs, Mladen Žujović ayant repris le commandement des unités armées en Bosnie occidentale à la suite de la mort d'Ilija Trifunović-Birčanin, Stevan Moljević devient de fait le premier conseiller politique de Draža Mihailović et le rédacteur en chef du journal Ravna Gora. Mais en 1943, le mouvement de la Ravna Gora se retrouve affaibli face à la résistance communiste qui pose les jalons du futur État yougoslave ${ }^{42}$.

Ayant perdu l'initiative politique, le mouvement de la Ravna Gora organise du 25 au 28 janvier 1944 dans le village de Ba un congrès visant à adopter un programme sur l'organisation de la nouvelle Yougoslavie ${ }^{43}$. Ce rassemblement apparaît comme une réponse à la Seconde session du Conseil antifasciste de la lutte de libération nationale de Yougoslavie (AVNOJ) dominée par les communistes qui s'est tenu en 1943 et a posé les fondements du nouvel État yougoslave, à savoir la création d'un État fédéral comprenant six républiques. Près de 300 délégués venus de tout le pays assistent au congrès de Ba. Ce dernier est l'occasion de réinsérer certains partis (Parti socialiste de Yougoslavie, Parti démocrate, etc.) d'avant-guerre dans le jeu politique, contre l'avis des principaux idéologues du mouvement de la Ravna Gora - Stevan Moljević et Dragiša Vasić, qui vouent une profonde aversion pour la classe politique yougoslave de l'entre-deux-guerres et auraient préféré un élargissement de leur structure plutôt que la création d'une nouvelle organisation politique. Afin d'améliorer l'image du mouvement et de renforcer son caractère démocratique, les représentants de certains partis politiques décident de s'unir tant que la guerre dure dans une coalition portant le nom de Communauté populaire démocratique yougoslave (Jugoslovenska demokratska narodna zajednica) et de s'associer au mouvement de la Ravna Gora.

Le Congrès prévoit dans sa résolution le renouvellement de l'État yougoslave et son élargissement à des territoires peuplés de Serbes, Croates et Slovènes. Le minimum de ce territoire devra correspondre à ce qu'avait demandé la délégation yougoslave à la Conférence de la paix après la Première Guerre mondiale ${ }^{44}$. La nouvelle Yougoslavie sera une monarchie parlementaire avec à sa tête le roi Petar II Karadjordjević (point 4-a de la résolution finale du congrès). L'État sera organisé sur une base fédérale comprenant trois unités : la Serbie, la Croatie et la Slovénie ${ }^{45}$. L'unité fédérale serbe comprendra tout le peuple serbe (point 4-c). Ce principe sera valide également pour la Croatie et la Slovénie. Le Congrès décide d'annuler toutes les modifications territoriales intervenues avant et pendant la guerre : la Banovine de Croatie, le démantèlement de la Yougoslavie par les forces d'occupation, la création de l'État croate indépendant (point $4-d)$. L'orientation yougoslave du Congrès correspond aux positions du gouvernement yougoslave en exil qui s'était prononcé pour la formation d'un État fédéral à trois unités (Serbie, Croatie, Slovénie). Mais si les idéologues du mouvement de la Ravna Gora acceptent cette orientation, c'est uniquement à la condition que les Serbes jouent le rôle dominant dans le nouvel État ${ }^{46}$. De surcroît, cette nouvelle Yougoslavie devra être un «État pur sans minorités nationales » ${ }^{47}$. Par ailleurs, le congrès dénonce l'action du Parti communiste de Yougoslavie qui a créé son armée et tente d'imposer sa dictature en Yougoslavie.

29 Au cours de ce congrès, Stevan Moljević s'est opposé à l'effacement du Comité central national (CNK) au profit d'une nouvelle organisation politique ${ }^{48}$. Il préconise toujours le rejet des acteurs politiques de l'entre-deux-guerres et souhaite que le mouvement prenne davantage ses distances à l'égard du gouvernement yougoslave en exil. Sa hantise est de voir ce qu'il a construit détruit par les représentants des partis politiques 
démocratiques. Il est en particulier sévèrement critiqué par le président du Parti socialiste de Yougoslavie, Živko Topalović, qui est à l'origine du congrès et représente les partis politiques démocratiques de Belgrade ${ }^{49}$. À l'issue du congrès, le Comité central national est élargi et Stevan Moljević en est nommé président. Sa position de second dans la hiérarchie du mouvement est ainsi confirmée. Le Mouvement de la Ravna Gora a adhéré à la nouvelle organisation politique (Jugoslovenska demokratska narodna zajednica) et est censé ne plus avoir d'activités politiques propres jusqu'à la libération du pays.

Si le congrès a affiché une dimension yougoslave, les principaux idéologues, Stevan Moljević et Dragisa Vasić, considèrent que le principal objectif de l'unification des pays serbes doit être mis au premier plan. En février 1944, ils prennent l'initiative de lancer un nouveau journal, ayant pour titre Serbité unifiée (Ujedinjeno srpstvo). Dans le premier numéro, paru le 2 avril 1944, cet objectif est rappelé dans l'article «Pour notre unification et notre unité » (« Za naše ujedinjenje i naše jedinstvo »), attribué à Stevan Moljevićc ${ }^{50}$. Selon lui, les intérêts spécifiquement serbes ont été oubliés entre 1918 et 1941. Le rétablissement de l'État yougoslave n'est possible que si l'ensemble des Serbes se trouvent réunis dans une seule entité politique.

La rupture avec le gouvernement yougoslave en exil intervient après la nomination d'Ivan Šubašić, dirigeant du Parti paysan croate (HSS), au poste de premier ministre le $1^{\text {er }}$ juin $1944^{51}$. Les forces de l'Armée yougoslave dans la patrie sont clairement désavouées par le roi, Petar II de Yougoslavie, le 12 septembre $1944^{52}$. En effet, ce dernier appelle les Serbes, les Croates et les Slovènes à s'unir et à rejoindre l'Armée de libération nationale sous le commandement du maréchal Tito. Le 21 octobre 1944, le commandement suprême de la JVuO prend la décision de se retirer vers la Bosnie, consécutivement à la libération de Belgrade par l'Armée rouge, entrée en Yougoslavie le 22 septembre. Au printemps 1945, l'armée de Draža Mihailović , affaiblie et sans ressources, arrive dans la région de la Bosanska Krajina. Tandis que Draža Mihailović souhaite reprendre le chemin de la Serbie, Stevan Moljević décide de rester dans la région où il a passé le plus clair de son existence. C'est à cette époque qu'il consigne ses notes sur son engagement pendant les années de guerre ${ }^{53}$.

Arrêté le 3 septembre 1945, il est ensuite transféré de Banja Luka à Belgrade où il est détenu. Draža Mihailović est arrêté le 13 mars 1946. Le procès contre les dirigeants du mouvement de la Ravna Gora et les membres serbes du gouvernement yougoslave en exil débute le 10 juin 1946. Stevan Moljević est accusé d'avoir occupé des fonctions importantes au sein du comité exécutif du Comité central national (CNK), correspondant à la direction politique du mouvement de la Ravna Gora en Yougoslavie. Le procureur lui reproche d'avoir participé à la prise de décision concernant tous les aspects de la vie de l'organisation en question dans le cadre de réunions avec Draža Mihailović, tant à propos des liens avec les occupants, qu'avec le gouvernement yougoslave en exil, mais également à propos des orientations de la propagande ${ }^{54}$. Il est accusé d'avoir dirigé la " propagande četnik » du printemps 1943 au printemps 1945 et d'avoir ainsi encouragé les assassinats de ceux qui soutenaient « la lutte de libération des peuples de Yougoslavie », ainsi que des Croates et des musulmans ${ }^{55}$. Le 15 juillet 1946, tandis que Draža Mihailović est condamné à mort, Stevan Moljević se voit condamner à vingt ans de prison et de travail forcé. Il est conduit dans la prison de Sremska Mitrovica où il décèdera de maladie à l'âge de 72 ans le 15 novembre $1959^{56}$. Plusieurs manuscrits ont été saisis par l'administration pénitentiaire portant en 
particulier sur l'histoire de la Bosnie-Herzégovine : «Le procès pour haute trahison de

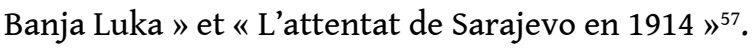

\section{Conclusion}

Stevan Moljević était avant tout un notable et un intellectuel de Banja Luka, attaché à la démocratie et aux alliés occidentaux de la Première guerre mondiale, la France et la Grande-Bretagne, comme en témoignent ses nombreuses initiatives dans le domaine culturel. Il ne connaît pas la Serbie qu'il découvre réellement en 1942 et n'est pas fortement impliqué dans les réseaux intellectuels et politiques actifs à Belgrade, malgré son investissement dans le Club culturel serbe à partir de 1939. Il défend en premier lieu les intérêts des Serbes de Bosnie-Herzégovine et plus particulièrement les Serbes de la région de la Bosanska Krajina. En août 1941, il est intégré à l'initiative de Dragiša Vasić et de Mladen Žujović, sans le savoir à l'époque, au comité exécutif du Comité central national du mouvement de la Ravna Gora dirigé par l'officier de l'armée yougoslave, Draža Mihailović. Ce n'est qu'en mai 1942, qu'il rencontre ce dernier et décide de rejoindre le mouvement lancé au printemps 1941. Entre-temps, il a publié un programme pour la réorganisation de l'État yougoslave prévoyant la formation d'une grande entité serbe qui rassemblerait l'ensemble du peuple serbe. Cet objectif s'inscrit dans la continuité des positions avancées par le Club culturel serbe et rejoint le projet de « Pays serbe » (Srpska zemlja) formulé en 1940, en réponse à l'établissement en août 1939 de la banovine de Croatie.

La dimension nouvelle vient de la proposition de procéder à des échanges de population entre Serbes et Croates. Cette proposition est liée à la guerre qui vient d'éclater, au démantèlement de la Yougoslavie et aux menaces physiques qui pèsent contre les Serbes, en particulier dans l'État croate indépendant (NDH). Elle ne saurait être isolée de ce contexte de crise propice à ce type de solutions ${ }^{58}$. Toutefois, certains membres du Club culturel serbe avaient déjà projeté des échanges ou expulsions de population avant la guerre dans le but de réduire les minorités nationales présentes sur le territoire yougoslave (Albanais, Hongrois, etc.).

En 1943, Moljević devient le premier conseiller politique du général Draža Mihailović, Dragiša Vasić ayant exprimé des divergences avec ces deux derniers après les revers militaires du printemps 1943. Il demeure fidèle aux alliés occidentaux, à savoir à la Grande-Bretagne et aux États-Unis. L'historiographie communiste a classé le mouvement de la Ravna Gora dans le camp des forces fascistes ayant collaboré avec les Occupants. Force est de constater que la réalité est plus complexe. Le projet de Stevan Moljević d'unification des Serbes dans une entité à l'intérieur de la Yougoslavie n'était guère envisageable, tant il réduisait la Croatie à une portion congrue. De surcroît, il n'était réalisable que dans la violence ${ }^{59}$. Le mouvement de la Ravna Gora ne disposait pas d'un appareil d'État pour réaliser ses objectifs ${ }^{60}$.

À la fin des années 1980, les idées de Stevan Moljević ont été exploitées par Vojislav Šešelj, fondateur du Mouvement četnik serbe (Srpski četnički pokret, SčP) en juin 1990. Elles ont été rattachées aux acteurs politiques serbes nationalistes et ont été présentées et discutées lors de procès au Tribunal pénal international pour l'ex-Yougoslavie ${ }^{61}$. Alors que son projet de Grande Serbie avait été formulé en pleine guerre en réaction à la destruction de la Yougoslavie par les Allemands et les Italiens, ainsi qu'à la violence visant les Serbes de la part des ustaše croates, ses idées ont été utilisées à la fin des 
années 1980 et au début des années 1990 dans le but d'établir les nouvelles «frontières occidentales » de la Serbie.

\section{NOTES}

1. Le récit officiel du régime communiste yougoslave a commencé à être critiqué au cours des années 1980 en premier lieu par des écrivains (Vuk Drašković, Jovan Radulović, etc.). En Serbie, l'historien V. Đuretić est le premier à questionner le monopole des partisans communistes de Tito sur la résistance opposée aux forces de l'Axe (Đuretić (Veselin), Saveznici i jugoslovenska ratna drama [Les Alliés et le drame yougoslave de la guerre], Beograd: Narodna knjiga / Balkanološki institut SANU, 1985). Depuis, les ouvrages parus dans l'immigration ont été réédités en Serbie et ont permis de compléter les sources et les regards sur les événements de la Seconde Guerre mondiale. Mais ce n'est qu'après 2000 que les manuels scolaires ont été révisés dans le sens d'une réhabilitation du mouvement de résistance royaliste (Voir Cvijic (Srdjan), «Swinging the Pendulum : World War II History, Politics, National Identity and Difficulties of Reconciliation in Croatia and Serbia ", Nationalities papers, 36 (4), 2008, p. 722). L'historiographie communiste visait plusieurs objectifs politiques : justifier et légitimer le rôle du Parti communiste yougoslave pendant la Seconde Guerre mondiale, présenter le mouvement des partisans comme le seul mouvement de résistance aux forces d'occupation, contribuer à l'entente entre les différentes nations et minorités dans le cadre d'un État fédéral complexe (ibid., p. 714).

2. Le mouvement de la Ravna Gora, qui est également désigné sous le nom moins officiel de "Mouvement četnik», est fondé par des officiers de l'Armée yougoslave ayant refusé de se rendre aux Allemands après la signature de la capitulation de la Yougoslavie en avril 1941. Il se structure autour de la personnalité du colonel Draža Mihailović (1893-1946) qui, commandant la Seconde armée en Bosnie-Herzégovine pendant la guerre d'avril 1941, se replie en Serbie occidentale avec ses hommes dans la région de la Ravna Gora, située entre les villes de Valjevo et Čačak. Ayant fait le choix de la résistance aux forces de l'Axe, Draža Mihailović fonde durant l'été 1941 un état-major embryonnaire sous le nom de Commandement des unités četniks de l'Armée yougoslave. Ces unités sont rebaptisées peu de temps après "Unités militaro-četniks » (Vojnočetnicki odredi). En novembre 1941, le gouvernement yougoslave en exil nomme Draža Mihailović commandant des forces patriotiques demeurées en Yougoslavie. Les forces četniks sont rebaptisées à cette occasion « Armée yougoslave dans la patrie " (Jugoslovenska vojska u Otadžbini, $\mathrm{JVuO}$ ) afin de souligner la continuité militaire et étatique avec la Yougoslavie d'avant-guerre. Le terme četnici pour désigner les soldats est abandonné dans les documents officiels du mouvement de la Ravna Gora mais est largement employé dans la population. En janvier 1942, Draža Mihailović est nommé ministre des Armées, de la Marine et de l'Aviation par le gouvernement yougoslave en exil

3. Les biographies de Dragoljub Mihailović se sont multipliées depuis le début des années 1990. L'auteur le plus productif à propos du mouvement de la Ravna Gora est sans conteste le publiciste et journaliste Miloslav Samardžić (1963-) dans le cadre de la maison d'édition Pogledi ou Novi pogledi. Sur le plan des études universitaires, Kosta Nikolić est l'auteur le plus prolifique : il est en particulier l'auteur d'un ouvrage en trois volumes: Nikolić (Kosta), Istorija ravnogorskog pokreta 1941-1945 [Histoire du mouvement de la Ravna Gora], Belgrade: Srpska reč, 1999, correspondant à sa thèse de doctorat. 
4. Voir par exemple: Greater Serbia : from ideology to aggression, Zagreb : Croatian Information Centre, 1993 ; Grmek (Mirko), Gjidara (Marc), Simac (Neven), Le nettoyage ethnique : documents historiques sur une idéologie serbe, Paris : Fayard, 1993.

5. Une seule monographie lui est consacrée: Todorović (Dragoje), Dr. Stevan Moljević: rečju, perom, delom i životom za Ujedinjeno Srpstvo [Stevan Moljević : pour la serbité unifiée par la parole, l'écriture, l'œuvre et la vie], Belgrade: Kalekom, 2000. La plupart des articles et ouvrages mentionnant le fameux texte de 1941 ne présentent pas son auteur et son cheminement intellectuel. Son nom ne figure pas dans l'Encyclopédie du peuple serbe, éditée en 2008 (Enciklopedija srpskog naroda, Belgrade : Zavod za udžbenike, 2008).

6. Le président du SKK, Slobodan Jovanović, est nommé chef du gouvernement yougoslave en exil en janvier 1942.

7. Todorović (Dragoje), op.cit., p. 11.

8. L'attentat est prévu à l'occasion de l'inauguration du parlement de la Bosnie-Herzégovine le 15 juin 1915.

9. Moljević (Stevan), «Žerajičev atentat », Razvitak, (2), 1936, p. 45. Texte cité par Todorović (Dragoje), op.cit., p. 13.

10. Todorović (Dragoje), op.cit., p. 16.

11. En 1916, 16 accusés furent condamnés à mort, 33 furent libérés faute de preuves suffisantes, les autres furent condamnés à des peines de prison de 5 à 20 ans. Mais sur l'intervention du roi d'Espagne, les 16 condamnés à mort virent leur peine commutée en 20 ans d'emprisonnement. Au cours de ce procès, la femme de Stevan Moljević, Branka, fut emprisonnée avec son fils âgé d'un an, Vladimir, pour avoir salué dans Banja Luka la colonne des accusés.

12. En 1936, la bibliothèque a reçu 300 ouvrages qu'elle a pu se procurer grâce à une aide du gouvernement français de 6000 francs (Todorović (Dragoje), op.cit., pp. 42-43)

13. Sa maison est située au numéro 5 de la rue du Prince Pavle (Todorović (Dragoje), op.cit., p. 42).

14. Le consul de France à Zagreb, M. Guéraud, assiste à l'ouverture officielle du cercle français à Prijedor, en compagnie du vice-consul, M. Varnier, ainsi que du consul polonais, M. Burda.

15. Ces activités sont rapportées dans la publication Otažbina (Todorović (Dragoje), op.cit., p. 43).

16. Todorović (Dragoje), op.cit., p. 68.

17. Stojan Protić, Miša Trifunović, Jaša Prodanović, Ljubomir Stojanović, Milan Grol, Slobodan Jovanović, etc.

18. Janićijević (Milosav), Stvaralačka inteligencija medjuratne Jugoslavije [L’intelligentsia créatrice de la Yougoslavie de l'entre-deux-guerres), Belgrade : Institut društvenih nauka, 1984, p. 125.

19. Nikolić (Kosta), "Dragiša Vasić : skica za portret nacionalnog revolucionara » [Dragiša Vasić : esquisse pour le portrait d'un révolutionnaire national], Istorija 20. veka, (1), 1997, p. 99.

20. Ses statuts sont validés parle ministère de l'Intérieur du Royaume de Yougoslavie le 15 janvier 1937. L'objectif de l'association est de «cultiver la culture serbe en dehors des partis politiques et de la vie politique ».

21. Dimić (Ljubodrag), « Srpski kulturni klub izmedju kulture i politike : prilog istoriji » [Le Club culturel serbe entre culture et politique : contribution à son histoire], Književnost, (9-10), 1993, p. 863.

22. Le premier numéro de Srpski glas paraît le 16 novembre 1939. Il paraîtra chaque jeudi jusqu'au 13 juin 1940 lorsque le gouvernement yougoslave l'interdira.

23. Parmi les conférences organisées en 1937 et 1938 : Slobodan Jovanović, "La nécessité de l'initiative privée dans les questions de culture nationale (07/02/1937), Vasa Čubrilović, «Le problème de la colonisation intérieure en Serbie du sud » (07/02/1937), Dragiša Vasić, «L'idée de Patrie et de justice sociale » (28/02/1937), Vladimir Ćorović, « La coordination du travail de nos associations culturelles et éducatives» (15/04/1937), Josif Mihajlović, «La situation en Macédoine » (10/05/1937), Slobodan Drašković, «A propos de la culture serbe » (26/05/1937), 
Radmilo Vučić, «Les chants populaires et la vie sociale contemporaine » (31/05/1937), Djoka Perin, «La nationalisation de la Voïvodine et de la Serbie du sud» (17/06/1937), Nikola Stojanović, «A propos du serbisme et du yougoslavisme » (14/11/1937), Ljubomir Pokorni, «Le lien spirituel entre l'armée et le peuple dans la guerre contemporaine » (22/11/1937), Nikola Djonović, «La situation au Monténégro » (29/11/1937), Mihajlo Konstantinović, « Les réglements constitutionnels sur l'éducation » (13/12/1937), Djoka Perin, «A propos de la nationalisation des musulmans de Bosnie-Herzégovine » (24/01/1938), Mehmed Begović, «A propos du problème musulman en Bosnie et Herzégovine » (07/02/1938), Slobodan Drašković, « La culture nationale et la jeunesse» (14/02/1938), Vasa Čubrilović, «Le problème religieux en Yougoslavie » (21/03/1938), Orestije Krstić « Le combat pour la terre en Serbie du sud» (04/04/1938), Slobodan Jovanović, «Confédération et fédération » (18/04/1938), Jovan Djordjević, « Nation, culture et Etat », (02/05/1938), Milan Petrović, « La situation en Voïvodine » (06/05/1938), etc. Voir Dimić (Ljubodrag), op.cit., p. 867.

24. Fils de Stefan Nemanja, fondateur de la dynastie des Nemanjić, Rastko s'est consacré à la vie religieuse et monacale sous le nom de Sava. Grâce à son action, l'Église orthodoxe serbe est devenue autocéphale en 1219. Il en devient le premier archevêque.

25. Selon Stevan Moljević, ces territoires forment une unité compacte de 1200000 habitants et un «mur vivant » qui sépare les Croates du nord de ceux de Bosnie centrale et d'Herzégovine occidentale. Todorović (Dragoje), op.cit., p. 96.

26. La nouvelle banovine de Croatie comprendra dans ses limites les districts de Derventa et de Gradačac retirés à la banovine du Vrbas.

27. La Banovine de Croatie comprend les banovines de la Save et de la Drave, les districts de Dubrovnik (Banovine de la Zeta), Derventa et Gradačac (Banovine de Vrbas), Travnik, Fojnica et Brčko (Banovine de la Drina), Šid et Ilok (Banovine du Danube).

28. «Sporazum ili nesporazum » [Accord ou désaccord], Srpski glas, (12), 01/02/40.

29. Jovanović (Slobodan), « Jugoslovenska misao » [La pensée yougoslave], Srpski glas, (8), 1940.

30. On peut retrouver les conceptions nationales des membres du Club culturel dans leur organe Srpski glas [La voix serbe] publié à partir de 1939. À propos de ce journal voir : Jovičić (Miodrag), Jako srpstvo-Jaka Jugoslavija: izbor članaka iz Srpskog glasa, organa Srpkog kulturnog kluba, 1939-1940 [Serbité forte-Yougoslavie forte: choix d'articles de Srpski glas, organe du Club culturel serbe), Beograd : Naučna knjiga, 1991.

31. Voir la traduction française parue dans Grmek (Mirko) et al., op.cit, p. 167.

32. Perin (Djoko), "Nacionalizovanje Vojvodine i Južne Srbije» [La nationalisation de la Voïvodine et de la Serbie du sud].

33. Selon les données fournies par Djoko Perin lors de sa conférence sur la « nationalisation de la Voïvodine et de la Serbie du sud ».

34. Les Bunjevci sont catholiques et habitent entre le Danube et la rivière Tisza. Originaires de Dalmatie et d'Herzégovine, ils se sont installés dans cette région au XVII ème $s$. pour fuir les incursions ottomanes. Plusieurs théories s'affrontent quant à l'appartenance des Bunjevci aux nations serbe et croate. À propos de la question des Bunjevci, voir Tosijević (Bojan), "Why Bunjevci Didn't Became a Nation : a Case Study », East Central Europe, 29 (1-2), 2002.

35. Dimić (Ljubodrag), op.cit., p. 865.

36. «Homogena Srbija » [Serbie homogène], 30.06.1941, repris dans Vesović (Milan), Nikolić (Kosta), Ujedinjene srpske zemlje: ravnogorski nacionalni program [Pays serbes unifiés: le programme national de la Ravna Gora],Belgrade : Vreme knjige, 1996, p. 190.

37. Cette analyse est partagée par les autres idéologues du mouvement de la Ravna Gora, Dragiša Vasić en particulier.

38. Todorović (Dragoje), op.cit., p. 135.

39. La responsabilité éditoriale en est confiée à Dragiša Vasić, qui s'occupera des huit premiers numéros avant d'exprimer sa discorde avec Stevan Moljević. 
40. Cité par Nikolić (Kosta), op.cit., t. 2, pp. 389-390.

41. Les premières divergences entre Dragiša Vasić et Draža Mihailović sont apparues au printemps 1943 après les défaites sur la Neretva et la Drina. Dragiša Vasić se montre critique entre autres par rapport à la lutte sans merci menée contre les partisans communistes au Monténégro, en Herzégovine et au Sandžak. Il formulera à plusieurs reprises ses critiques quant aux faiblesses du mouvement de la Ravna Gora dans des courriers adressés à Draža Mihailović. Il dénoncera le fait que les soldats gaspillent leurs munitions lors de fêtes et mariages, qu'ils s'adonnent à des actions sanguinaires en égorgeant des ennemis plutôt qu'en les fusillant, etc. Nikolić (Kosta), art.cit., p. 103 ; Milovanović (Nikola), Dragiša Vasić: od gradjanskog buntovnika do kontrarevolucionara [Dragiša Vasić : du citoyen révolté au contre-révolutionnaire], Belgrade : Nova knjiga, 1986, p. 35.

42. Début 1943, les partisans communistes ont subi des offensives de la part des forces d'occupation allemandes et italiennes, auxquelles ont également participé des forces liées au mouvement de la Ravna Gora : les opérations Weiss 1 et Weiss 2 avaient pour objectif de réduire à néant le mouvement de résistance communiste. Une troisième phase conclue entre Allemands et Italiens devait aboutir au désarmement des forces répondant au commandement de Draža Mihailović. Néanmoins, les Italiens ont refusé le désarmement des forces armées serbes estimant qu'il n'y avait pas à craindre un débarquement des Alliés le long de la côte adriatique. Ils estimaient que les Allemands souhaitaient prendre le contrôle du littoral adriatique et de l'arrière-pays situé en Bosnie et en Herzégovine à leur dépens (Nikolić (Kosta), Italijanska vojska $i$ četnici u drugom svetskom ratu u Jugoslaviji 1941-1943 [L'armée italienne et les Tchetniks durant la Seconde Guerre mondiale en Yougoslavie 1941-1943], Belgrade, ISI, 2009, p. 414). Le 14 mai, l'opération Schwartz est lancée par le général Löhr: elle a pour objectif de désarmer et d'emprisonner les forces armées qui n'appartiennent pas aux forces régulières allemandes, italiennes et croates. Au cours de ces opérations, les forces royalistes serbes subissent de nombreux revers et les partisans communistes, malgré de nombreuses pertes humaines, parviennent à s'extirper de l'étau allemand. Voir aussi Vasic (Roland), Mihailović entre révolution et restauration: Yougoslavie 1941-1946, Paris : L'Harmattan, 2009, pp. 146-154.

43. Mais cette initiative est trop tardive. Les alliés, et en particulier les britanniques ne considèrent plus l'Armée yougoslave dans la patrie comme un facteur militaire significatif. Lors de la conférence de Téhéran (28 novembre- $1^{\text {er }}$ décembre 1943), il a été décidé d'appuyer le mouvement des partisans communistes au détriment des forces sous le commandement de Draža Mihailović apparaissant plus proches de la collaboration avec les Allemands que de la lutte armée contre eux (Nikolić (Kosta), Istorija ravnogorskog pokreta (op.cit.), t. 3, pp. 198-204).

44. À l'époque, la délégation yougoslave avait demandé un élargissement du Royaume des Serbes, Croates et Slovènes aux régions de Skadar (Albanie), Timisoara (Roumanie), Kjustendil et Vidin (Bulgarie), Szeged et Pecuj (Hongrie), Istrie, Rijeka (Italie) et Carinthie (Autriche).

45. Au cours des discussions préparatoires, Živko Topalović a proposé la création de quatre unités fédérales en proposant que la Bosnie-Herzégovine soit la quatrième unité. Mais il a rencontré une ferme opposition de la part de Stevan Moljević, ainsi que de Dragiša Vasić (Topalović (Živko), Borba za budućnost Jugoslavije - Combat pour l'avenir de la Yougoslavie, Paris / London : Sindikalist, 1967, pp. 160-161).

46. Nikolić (Kosta), art.cit., p. 67.

47. Selon les décisions du Congrès de Ba présentées dans le journal Pomoravlje au printemps 1944. Articles de presse reproduits dans Matić (Milan B.), Ravnogorska ideja u štampi i propagandi četnickog pokreta u Srbiji 1941-1944 [L'idée de la Ravna Gora dans la presse et la propagande du mouvement četnik en Serbie, 1941-1944], Belgrade : Institut za savremenu istoriju, 1995, pp. 198-204.

48. Topalović (Živko), op.cit. 
49. Topalović (Živko), op.cit., pp. 162-163. Stevan Moljević n'était pas très favorable à la tenue de ce congrès (ibid., p. 171).

50. Todorović (Dragoje), op.cit., pp. 183-184.

51. La décision est prise lors de la réunion plénière du Comité central national qui se tient du 20 au 23 juillet 1944.

52. Pavlowitch (Stevan K.), Hitler's New Disorder: the Second World war in Yugoslavia, London: Hurst, 2008, p. 232. Depuis la fin 1943, le mouvement des Partisans communistes est le principal bénéficiaire de l'aide britannique. Churchill a désormais décidé de s'appuyer sur Tito, tout en pensant pouvoir préserver la monarchie. Au printemps 1944, des pressions sont ensuite exercées sur le jeune roi, Petar II, pour qu'il forme un nouveau gouvernement susceptible de s'entendre avec Tito. Le changement est intervenu après la conférence de Téhéran qui s'est tenue du 28 novembre au $1^{\text {er }}$ décembre 1943. Le Foreign Office estimait que les forces de Mihailović étaient incapables de contribuer significativement à la libération de la Yougoslavie. Voir Nikolić (Kosta), Istorija ravnogorskog pokreta (op.cit.), t. 3, pp. 198-204.

53. Ses notes sont intitulées « Ma voie " (Moj put).

54. Zečević (Miodrag), Dokumenta sa suđenja ravnogorskom pokretu : 10 juni - 15 juli 1946 godine. Prva knjiga [Documents du procès contre le mouvement de la Ravna Gora : 10 juin-15 juillet 1946 . Tome premier], Belgrade : SUBNOR Jugoslavije, 2001, pp. 144-145.

55. Ibid., p. 145.

56. Todorović (Dragoje), op.cit., p. 273. Il sera enterré dans le cimetière de la prison de Sremska Mitrovica où son corps reposera jusqu'en 1977. Ses ossements seront ensuite transférés à Belgrade.

57. Ibid., p. 274.

58. Barutciski (Mickael), «Lausanne Revisited : Population Exchanges in International Law and Policy ", inHirschon (Renée), ed., Crossing the Aegean: an Appraisal of the 1923 Compulsory Population Exchange Between Greece and Turkey, London : Berghahn Books, 2003, p. 25.

59. Vesović (Milan), Nikolić (Kosta), op.cit., p.149.

60. À ce propos, voir Dulić (Tomislav), «Mass killing in the Independent State of Croatia, 1941-1945 : a Case for Comparative Research », Journal of Genocide Research, 8 (3), 2006, p. 267.

61. Dans le cadre entre autres des procès IT-94-1-T (Duško Tadić), IT-03-67-T (Vojislav Šešelj), IT-02-54 (Slobodan Milošević).

\section{RÉSUMÉS}

L'article retrace le parcours de Stevan Moljević, l'un des principaux idéologues du mouvement de la Ravna Gora dirigé entre 1941 et 1946 par Dragoljub (dit Draža) Mihailović. Stevan Moljević, avocat de Banja Luka, a joué un rôle significatif dans la vie intellectuelle et culturelle de cette ville pendant les années 1920 et 1930. En 1939, il s'investit dans le Club culturel serbe contestant l'établissement d'une entité croate au sein du royaume de Yougoslavie, la banovine de Croatie. En 1942, il rejoint le commandement suprême de l'Armée yougoslave dans la patrie, ainsi que la direction du mouvement de la Ravna Gora, aile politique de cette première. Il se prononce pour la création d'une grande Serbie dans une grande Yougoslavie comprenant trois unités fédérales : la Serbie, la Croatie et la Slovénie. L'article replace les idées de Stevan Moljević dans le contexte de l'époque en question. 
This article traces the course of Stevan Moljević, one of the main ideologues of the movement of the Ravna Gora conducted by Dragoljub (called Draža) Mihailović between 1941 and 1946. Stevan Moljević, lawyer in Banja Luka, has played a significant role in the intellectual and cultural life of this city during the 1920s and 1930s. In 1939, he became a member of the Serbian Cultural Club challenging the establishment of a Croat entity within the Kingdom of Yugoslavia, the banovina of Croatia. In 1942 he joined the Supreme Command of the Yugoslav Army in the fatherland, and the direction of the movement of the Ravna Gora, the political wing of the former. During the war, he advocates the creation of a Greater Serbia in a Greater Yugoslavia with three federal units: Serbia, Croatia and Slovenia. The paper puts the ideas of Stevan Moljević in the context of that period.

\section{INDEX}

Keywords : Dragiša Vasić, Dragoljub (Draža) Mihailović, population exchange, Serbia, Stevan Moljević, World War II

Mots-clés : Dragiša Vasić, Dragoljub (Draža) Mihailović, échanges de population, Seconde Guerre mondiale, Serbie, Stevan Moljević

Index géographique : Banja Luka, Bosanska Krajina, Bosnie-Herzégovine, Sarajevo, Serbie, Yougoslavie

\section{AUTEUR}

\section{YVES TOMIĆ}

